\title{
BMJ Open Women's acceptability of and experience with primary human papillomavirus testing for cervix screening: HPV FOCAL trial cross-sectional online survey results
}

\author{
Laurie W Smith (D) , ${ }^{1,2}$ C Sarai Racey, ${ }^{2,3}$ Lovedeep Gondara, ${ }^{4}$ Mel Krajden, ${ }^{3,5}$ \\ Marette Lee, ${ }^{3,6}$ Ruth Elwood Martin, ${ }^{3}$ Gavin Stuart, ${ }^{3}$ Stuart Peacock, ${ }^{1,7}$ \\ Andrew J Coldman, ${ }^{1}$ Eduardo L Franco (D) , ${ }^{8}$ Dirk van Niekerk, ${ }^{3,6}$ \\ Gina S Ogilvie (iD ${ }^{2,3}$
}

To cite: Smith LW, Racey CS, Gondara $\mathrm{L}$, et al. Women's acceptability of and experience with primary human papillomavirus testing for cervix screening: HPV FOCAL trial cross-sectional online survey results. BMJ Open 2021;11:e052084. doi:10.1136/ bmjopen-2021-052084

- Prepublication history and additional supplemental material for this paper are available online. To view these files, please visit the journal online (http://dx.doi.org/10.1136/ bmjopen-2021-052084).

Received 06 April 2021 Accepted 20 September 2021

Check for updates

(C) Author(s) (or their employer(s)) 2021. Re-use permitted under CC BY-NC. No commercial re-use. See rights and permissions. Published by BMJ.

For numbered affiliations see end of article.

Correspondence to

Ms Laurie W Smith;

laurie.smith@bccancer.bc.ca

\section{ABSTRACT}

Objective To study participant's acceptability of and attitudes towards human papillomavirus (HPV) testing compared with cytology for cervical cancer screening and what impact having an HPV positive result may have in future acceptability of screening.

Design Cross-sectional online survey of clinical trial participants.

Setting Primary care, population-based Cervix Screening Program, British Columbia, Canada.

Participants A total of 5532 participants from the HPV FOCAL trial, in which women received HPV and cytology testing at study exit, were included in the analysis. Median age was 54 years. The median time of survey completion was 3 years after trial exit.

Outcome measures Acceptability of HPV testing for primary cervical cancer screening (primary); attitudes and patient perceptions towards HPV testing and receipt of HPV positive screen results (secondary).

Results Most respondents (63\%) were accepting of HPV testing, with the majority $(69 \%)$ accepting screening to begin at age 30 years with HPV testing. Only half of participants (54\%) were accepting of an extended screening interval of 4-5 years. In multivariable logistic regression, women who received an HPV positive screen test result during the trial $(\mathrm{OR}=1.4195 \% \mathrm{Cl} 1.11$ to 1.80$)$ or were older $(\mathrm{OR}=1.01,95 \% \mathrm{Cl} 1.00$ to 1.02$)$ were more likely to report HPV testing as acceptable.

Conclusions In this evaluation of acceptability and attitudes regarding HPV testing for cervix screening, most are accepting of HPV testing for screening; however, findings indicate heterogeneity in concerns and experiences surrounding HPV testing and receipt of HPV positive results. These findings provide insights for the development of education, information and communication strategies during implementation of HPVbased cervical cancer screening.

Trial registration numbers ISRCTN79347302 and NCT00461760.

\section{INTRODUCTION}

It is well established that persistent infection with an oncogenic strain of the human papillomavirus (HPV), the most common

\section{STRENGTHS AND LIMITATIONS OF THIS STUDY}

$\Rightarrow$ Measures of acceptability and patient perceptions of human papillomavirus (HPV) testing within a primary cervix screening programme.

$\Rightarrow$ Reports on acceptability of increased screening interval and delayed onset of screening initiation, and impact of a positive HPV test result.

$\Rightarrow$ Recommendations for key health promotion messaging to address potential barriers to HPV testing for primary cervical screening.

$\Rightarrow$ Limitations include that participants were recruited from a large clinical trial on HPV testing for cervical cancer screening and may not be representative of the general screening population.

sexually transmitted infection (STI) around the world, is the causative agent for most cervical cancers. ${ }^{12}$ There is a robust body of evidence regarding the superior performance of HPV versus cytology screening in detection of cervical intraepithelial neoplasia grade two or worse and greater protection against cervical cancer. ${ }^{3-5}$ As such, several countries around the world have implemented primary HPV testing for cervical cancer screening, including Australia, the Netherlands and the UK, with many other jurisdictions in various planning stages for HPV-based screening implementation. A shift to an HPV-based screening approach results in different programme guidelines and, thus, a different experience for the person undergoing screening. The very high negative predictive value of HPV testing permits the interval between screens to be extended to 5 or more years compared with cytology testing, recommended every 2-3 years in most jurisdictions. ${ }^{5-7}$ Due to high prevalence and regression rates of HPV infection in younger women, HPV-based screening may 
not be recommended until $25-30$ years of age.$^{56}$ In addition, being screened for cervical cancer with a test for an STI can result in anxiety and concern for those undergoing screening. ${ }^{8} 9$

With such a transformative change in what is arguably a well-established screening paradigm, it is crucial to examine women's readiness or acceptance of HPV testing compared with cytology testing for screening to ensure engagement in screening is not hampered by a change in technology or guidelines. This unintended consequence was illustrated in Australia, prior to the change in the national programme from cytology to HPV screening, when a 2017 petition opposing the changes garnered 70000 signatures. ${ }^{10}$ Respondents to the Australian survey indicated concerns about such things as the extended interval and missing cancer cases in younger women as a result of the programme change. ${ }^{10}$ Despite enhancements to screening efficacy and safety, a successful change in technology requires acceptance by those who undergo screening. Anticipating women's questions and concerns prior to implementation of programme changes can mitigate resistance to change and assist in the design of targeted education strategies.

This analysis is of the 48-month exit survey for the Human Papillomavirus For Cervical Cancer Screening Trial (HPV FOCAL). HPV FOCAL is currently the only North American trial comparing primary HPV testing to cytology (liquid based) for screening within an organised programme, which also provides us with the unique opportunity to assess women's experiences with HPV testing in a population-based programme setting. The primary objective of this analysis was to explore participant's acceptability of and attitudes towards HPV testing compared with cytology for cervical cancer screening and what impact having an HPV positive result may play in future acceptability of screening.

\section{METHODS}

\section{Participants}

Survey participants were recruited through the HPV FOCAL trial, a publicly funded randomised control trial comparing primary HPV testing every 4 years (HPV arm) to liquid-based cytology testing (cytology arm) every 2 years for cervical cancer screening. HPV FOCAL recruited women, 25-65 years of age, from two largely metro areas in British Columbia who were due for cervical cancer screening from 2008 to 2012. Participants of the HPV FOCAL trial were engaged in cervical cancer screening through a large population-based screening programme and representative of women at average risk of cervical cancer in North America. ${ }^{11}$ Trial design and primary outcome results have been previously described in detail. ${ }^{311-14}$ Participants were provided with information on HPV, HPV testing (including differences between Pap and HPV testing and the reasons behind an extended interval between negative HPV screens) and cervical cancer on enrolment and throughout the trial follow-up period. A total of 9552 women were randomised to the HPV arm and 9457 women to the cytology arm. Women from both the HPV and cytology arms completed trial exit screening between 2012 and 2016, where they received HPV and cytology co-testing at the exit screen. Results were provided to their primary care provider, who then conveyed them to the participants. From August 2017 to February 2018, women from both arms who had attended the 48-month exit screen were invited to complete the online exit survey (figure 1).

\section{HPV FOCAL exit survey}

The survey included 26 items that asked participants about HPV knowledge and information seeking before and during the study, acceptability of HPV testing, willingness to increase the screening interval, commencement screening age for HPV testing, attitudes and concerns about test positive results and communication needs around screening results, in addition to demographic details (online supplemental file). Reponses included 7-point and 5-point Likert scales, and survey responses were linked to HPV FOCAL trial screen test results. Survey items were based on previous HPV FOCAL surveys assessing HPV testing acceptance. ${ }^{15}$ The survey was distributed and managed using the web-based platform of Fluidsurveys (www.fluidsurveys.com). The survey was pilot tested and revised for face and content validity with approximately 20 women, aged 30 years and above prior to distribution to FOCAL participants.

\section{Patient and public involvement}

Patient concerns and questions raised during the trial period identified the need for the study, but patients were not involved in the construction of the survey. ${ }^{16}$ However, a sample of women who undergo cervical cancer screening in $\mathrm{BC}$ were involved in pilot testing of the survey for the purposes of face validity and survey flow and logistics. Based on this feedback, revisions were made to the survey to clarify wording of questions and format of layout.

\section{Response rate and inclusion criteria}

Participants from the HPV FOCAL trial from both the HPV and cytology arms who had completed their study 48-month exit screen, had indicated consent to be contacted for future research and for whom email addresses were available were eligible for survey invitation. The invite to complete the survey was sent via email, with one reminder sent a month later for those who had not initiated or completed the survey. Participants were provided with a unique study identifier to access the survey and no personal identifiers were captured during survey completion. Participants were informed they had the option to complete none, some, or all of the survey with completion of survey questions as indication of consent.

Survey completeness was reviewed, and duplicate surveys, where the same woman completed all or some 


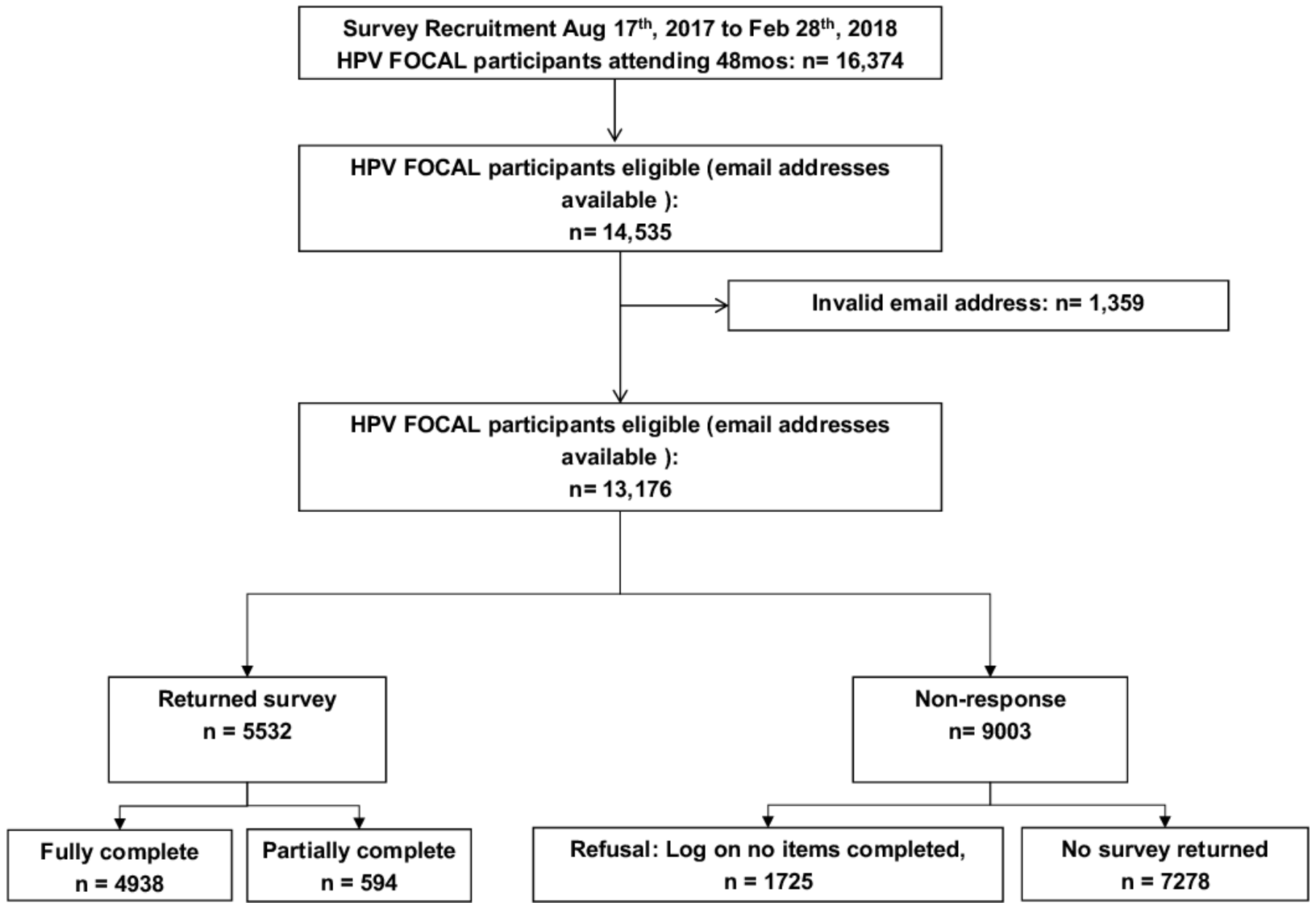

Figure 1 Study flow chart and participant disposition. HPV FOCAL, Human Papillomavirus For Cervical Cancer Screening Trial.

of the survey more than once, were identified. For those with a duplicate entry, the first complete survey was used in the analysis with all other survey attempts discarded. Response rate (\%) was the sum of completed surveys plus partial surveys, divided by the number of invitations sent to eligible valid email addresses, as per the American Association for Public Opinion Research guidelines. ${ }^{17}$ Non-response included: refusals (clicked the survey link, but did not complete consent or any items), and those assumed eligible with no response received. Email addresses that were undeliverable were considered invalid and not included in the analysis.

\section{Statistical analysis}

Survey respondents were compared with HPV FOCAL trial participants on age, study arm, and location of trial recruitment to explore if survey sample was representative of FOCAL trial population.

Our main outcome of HPV testing acceptability was assessed by response to the question 'having an HPV test instead of a Pap to screen for cervical cancer is acceptable to me', which was dichotomised from a 5-point Likert scale, with those reporting strongly agree or agree, categorised as 'accepting', and those reporting neutral, don't know, disagree and strongly disagree as 'not accepting' of
HPV testing. This categorisation was chosen to capture those who were truly accepting and biased towards the null. Only complete surveys were included, with those who were missing or preferred not to answer excluded. Participants were classified as being HPV positive, if they received an HPV positive screening result at any point during their participation in the HPV FOCAL trial; otherwise, a participant was classified as HPV negative.

Bivariable analysis explored differences in acceptability of HPV testing based on demographic factors such as age, income and education, in addition to HPV screening test result, and length of time since study exit. Factors shown to be potentially associated with acceptability, such as HPV screening starting at 30 years of age and increased screening interval, were also examined. ${ }^{161819}$

Sociodemographics and attitudes towards HPV testing were explored descriptively with $\chi^{2}$ for categorical variables and median score test for continuous variables.

Multivariate logistic regression was used to explore the association of the acceptability of HPV testing with a priori identified confounding variables that reached $\mathrm{p} \leq 0.2$ in bivariable analysis. Level of significance was 0.05 . All statistical analyses were performed in SAS V.9.4 and R V.4.02. 
Table 1 Demographic characteristics of respondents and bivariable analysis of acceptability of HPV testing

\begin{tabular}{|c|c|c|c|c|}
\hline & \multirow[b]{2}{*}{ Total (N) } & \multicolumn{3}{|c|}{$\begin{array}{l}\text { Having an HPV test instead of a Pap smear to screen for } \\
\text { cervical cancer is acceptable to me }\end{array}$} \\
\hline & & $\begin{array}{l}\text { Not acceptable } \\
\text { (disagree/neutral/don't } \\
\text { know), n (\%) }\end{array}$ & Acceptable (agree), n (\%) & $P$ value \\
\hline Total (N) & 5336 & 1993 (37.4) & $3343(62.6)$ & \\
\hline Median (IQR) & 5336 & 53.35 (45.35-61.19) & $54.23(45.64-61.72)$ & 0.051 \\
\hline Living with a partner & 3806 & $1438(78.7)$ & 2368 (76.6) & 0.095 \\
\hline Living without a partner & 1115 & $390(21.3)$ & $725(23.4)$ & \\
\hline \multicolumn{5}{|l|}{ Education† } \\
\hline Complete college or higher & 3317 & $1250(68.5)$ & 2067 (66.7) & 0.221 \\
\hline$\$ 75000$ or more & 3014 & $1126(68.0)$ & $1888(66.0)$ & \\
\hline \multicolumn{5}{|l|}{ HPV testing status during FOCAL trial } \\
\hline Never tested HPV positive & 4937 & $1867(93.7)$ & $3070(91.8)$ & $0.015^{\star}$ \\
\hline At least one HPV positive result & 399 & $126(6.3)$ & $273(8.2)$ & \\
\hline \multicolumn{5}{|l|}{ Time since exit from FOCAL trial, years } \\
\hline Median (IQR) & 5336 & $3.09(2.26-3.91)$ & $3.04(2.23,-3.93)$ & 0.616 \\
\hline \multicolumn{5}{|c|}{ I would be willing to have an HPV test every $4-5$ years instead of a Pap every 3 years $†$} \\
\hline Agree & 2858 & $386(19.5)$ & $2472(74.2)$ & $<0.001^{*}$ \\
\hline Disagree & 1096 & $744(37.6)$ & $352(10.6)$ & \\
\hline \multicolumn{5}{|c|}{$\begin{array}{l}\text { If cervical cancer screening was to occur every } 4 \text { or } 5 \text { years, instead of every } 3 \text { years, I would be less likely to visit my } \\
\text { healthcare provider for other health reasons. }\end{array}$} \\
\hline Agree & 1062 & $405(20.4)$ & $657(19.7)$ & 0.814 \\
\hline Disagree & 3517 & $1303(65.7)$ & $2214(66.4)$ & \\
\hline Neutral & 735 & $274(13.8)$ & $461(13.8)$ & \\
\hline
\end{tabular}

*Significant to $p<0.05$.

†Missing values up to $5336=$ not reported or prefer not to answer.

HPV, human papillomavirus; HPV FOCAL, Human Papillomavirus For Cervical Cancer Screening Trial.

\section{RESULTS}

Survey invites were administered from August 2017 to February 2018. A total of 14535 participants from both the HPV and cytology arms in HPV FOCAL trial were identified as eligible to receive 48-month exit online survey, of which 13176 were delivered to a valid email address (figure 1). There were 5532 surveys completed, of which 4938 were fully and 594 partially completed.

\section{Characteristics of respondents}

The median age of participants completing the survey was 54 years (IQR: 46-62) (table 1). The median time of survey completion was 3 years after study exit. The majority of respondents $(67 \%)$ had completed college or higher education and $77 \%$ reported living with a partner. Survey respondents were comparable with HPV FOCAL trial participants based on study arm, age at HPV FOCAL trial enrolment and geographical location. Survey respondents and non-respondents were comparable by study arm and marital status, but those who responded to the survey were slightly older than non-responders (median of 51 years vs 49 years), although the difference was not clinically significant. 
Table 2 Multivariate analysis of predictors for participants who are accepting of HPV testing instead of Pap testing for cervical cancer screening

\begin{tabular}{lll}
\hline Variable & OR (95\% Cl) & P value \\
\hline Age at survey completion (years) & 1.01 (1.00 to 1.02$)$ & 0.01 \\
\hline Received HPV positive results during study (yes vs no) & $1.41(1.11$ to 1.80$)$ & 0.005 \\
\hline Education (incomplete postsecondary or less vs complete college or higher) & $1.06(0.93$ to 1.21$)$ & 0.41 \\
\hline Marital status (living without a partner vs living with a partner) & $1.04(0.88$ to 1.22$)$ & 0.67 \\
\hline Income (\$75000 or more vs less than \$75 000) & $0.97(0.84$ to 1.12$)$ & 0.68 \\
\hline HPV, human papillomavirus. & &
\end{tabular}

\section{Acceptability of HPV testing for screening}

Overall, $63 \%$ of survey respondents agreed or strongly agreed that HPV testing for cervical cancer screening was acceptable, with $37 \%$ not agreeing (11\% disagree, $16 \%$ neutral, $10 \%$ don't know) (table 1). There were no significant associations between acceptability of HPV testing and marital partnership status, time since study exit, education or income. Women who received an HPV positive result at any point during the HPV FOCAL trial and who were older were more accepting of HPV testing compared with those who remained HPV negative during trial participation.

In multivariate analysis, women who reported HPV testing as acceptable were more likely to have received an HPV positive screen test result at some point during the trial (OR 1.41, 95\% CI 1.11 to $1.80, \mathrm{p}=0.005)$ and were older (OR 1.01, 95\% CI 1.00 to $1.02, \mathrm{p}=0.01$ ) (table 2).

Over half of respondents (54\%) agreed with the statement 'I would be willing to have an HPV test every 4-5 years instead of a Pap every 3 years'. There was a significant difference in acceptability of an extended screening interval between those who reported being accepting of HPV testing compared with those who were not accepting. Overall, $69 \%$ responded that HPV testing starting at age 30 years was acceptable, with over $80 \%$ of those who were accepting of HPV testing reporting agreement with a higher screening age (30 years or over) compared with Pap testing (table 1).

In addition, $66 \%$ of respondents reported that an extended screening interval would not result in less visits to their healthcare provider for other medical reasons, indicating that despite the extended interval recommended with HPV-based screening, women would continue to see their providers for medical reasons as needed.

\section{Attitudes towards an HPV positive test result}

Survey respondents were asked to rate their level of agreement with a variety of statements regarding attitudes surrounding receipt of HPV positive results (table 3). Women were asked if they would be more concerned about receiving a positive HPV test or an abnormal Pap result, for which most respondents $(73 \%)$ reported that both screening outcomes would concern them equally. However, those that reported HPV testing to be acceptable reported that an HPV positive result would concern them more (14\%) than an abnormal Pap. This was statistically different compared with those who were not accepting of HPV testing, who responded that abnormal Pap test results would concern them more.

Most respondents who were not accepting of HPV testing indicated that having a sexually acquired infection would concern them differently than having abnormal Pap results. The relationship between level of agreement with HPV testing acceptability and one's level of concern about having a sexually acquired infection was significant. Regardless of a respondent's reported HPV testing acceptability, most respondents felt it important to them to know who gave them HPV and when they acquired HPV (71\% and $78 \%$, respectively). Most respondents indicated they disagreed or were neutral regarding feeling judged for having HPV, and there was no significant relationship between feeling judged and level of agreement with HPV testing. More respondents who were accepting of HPV testing indicated they would feel comfortable telling their partner if they had HPV, which was in contrast to those who were not accepting of HPV testing; these differences indicated a significant relationship between HPV testing acceptance and comfort disclosing HPV status to a partner. Regardless of level of agreement with HPV testing, most respondents $(79 \%)$ indicated they would feel concerned about transmitting HPV to their partner(s). More respondents who were accepting of HPV testing would feel confident in the recommendations from their provider for the management of their HPV positive results. There was a significant association between level of agreement with HPV testing and degree of confidence with provider recommendations.

\section{Sources of information for HPV testing and screening}

Overall, the most reported important sources of information for all respondents were healthcare providers and BC Cancer, the agency that is responsible for the cervix screening programme in British Columbia (table 4). Further, those that are accepting of HPV testing were more likely to look to their healthcare providers and BC Cancer as important sources of information than those not accepting. Regardless of one's level of agreement with HPV testing for screening, friends and family or social media were not as important as healthcare providers and BC Cancer for sources of information. 
Table 3 Experiences receiving HPV positive results

\begin{tabular}{|c|c|c|c|c|}
\hline & \multicolumn{4}{|c|}{ HPV testing instead of Pap testing for cervical cancer: } \\
\hline & Total (N) & Not acceptable, n (\%) & Acceptable, n (\%) & $P$ value \\
\hline & 5336 & $1993(36.0)$ & $3343(60.4)$ & \\
\hline Being told I have abnormal Pap test results & 668 & $274(14.0)$ & $394(11.9)$ & $<0.001^{*}$ \\
\hline Being told I have HPV & 683 & $197(10.0)$ & $486(14.7)$ & \\
\hline Neither would concern me & 67 & $26(1.3)$ & $41(1.2)$ & \\
\hline \multicolumn{5}{|c|}{ Having an infection that is sexually acquired (HPV) does not concern me any differently than abnormal Pap results would $\dagger$} \\
\hline Agree & 1412 & $442(24.8)$ & $970(31.5)$ & $<0.001^{*}$ \\
\hline Disagree & 2465 & $974(54.6)$ & $1491(48.4)$ & \\
\hline Neutral & 987 & $369(20.7)$ & $618(20.1)$ & \\
\hline Neutral & 859 & $340(19.1)$ & $519(16.8)$ & \\
\hline \multicolumn{5}{|c|}{ It would be important for me to know when I got HPV } \\
\hline Agree & 3795 & $1372(76.9)$ & $2423(78.3)$ & 0.131 \\
\hline Disagree & 432 & $151(8.5)$ & $281(9.1)$ & \\
\hline Neutral & 650 & $260(14.6)$ & $390(12.6)$ & \\
\hline \multicolumn{5}{|l|}{ I think people would judge me for having HPV† } \\
\hline Agree & 1775 & $663(37.4)$ & $1112(36.0)$ & 0.307 \\
\hline Disagree & 1419 & $495(27.9)$ & $924(30.0)$ & \\
\hline Neutral & 1666 & $617(34.8)$ & $1049(34.0)$ & \\
\hline Disagree & 3825 & $1400(78.7)$ & $2425(79.0)$ & \\
\hline Neutral & 509 & $205(11.5)$ & $304(9.9)$ & \\
\hline \multicolumn{5}{|c|}{ Being HPV positive would not affect my relationship with my partner† } \\
\hline Agree & 1249 & $445(25.1)$ & $804(26.3)$ & $0.035^{*}$ \\
\hline Disagree & 2003 & $708(39.9)$ & $1295(42.3)$ & \\
\hline Neutral & 1584 & $622(35.0)$ & $962(31.4)$ & \\
\hline \multicolumn{5}{|c|}{ Having HPV would not cause me any concern about cervical cancer† } \\
\hline Agree & 181 & $52(2.9)$ & $129(4.2)$ & $0.012^{*}$ \\
\hline Disagree & 4112 & $1499(84.1)$ & $2613(84.8)$ & \\
\hline Neutral & 569 & $231(13.0)$ & $338(11.0)$ & \\
\hline \multicolumn{5}{|c|}{ I would feel confident in the recommendations from my healthcare provider for follow-up of my HPV positive result $†$} \\
\hline Agree & 3876 & $1323(74.0)$ & $2553(82.5)$ & $<0.001^{*}$ \\
\hline Disagree & 226 & $97(5.4)$ & $129(4.2)$ & \\
\hline Neutral & 780 & $369(20.6)$ & $411(13.3)$ & \\
\hline
\end{tabular}

*Significant to $\mathrm{p}<0.05$.

†Missing values up to $5336=$ not reported or prefer not to answer.

HPV, human papillomavirus. 
Table 4 Important sources of HPV information

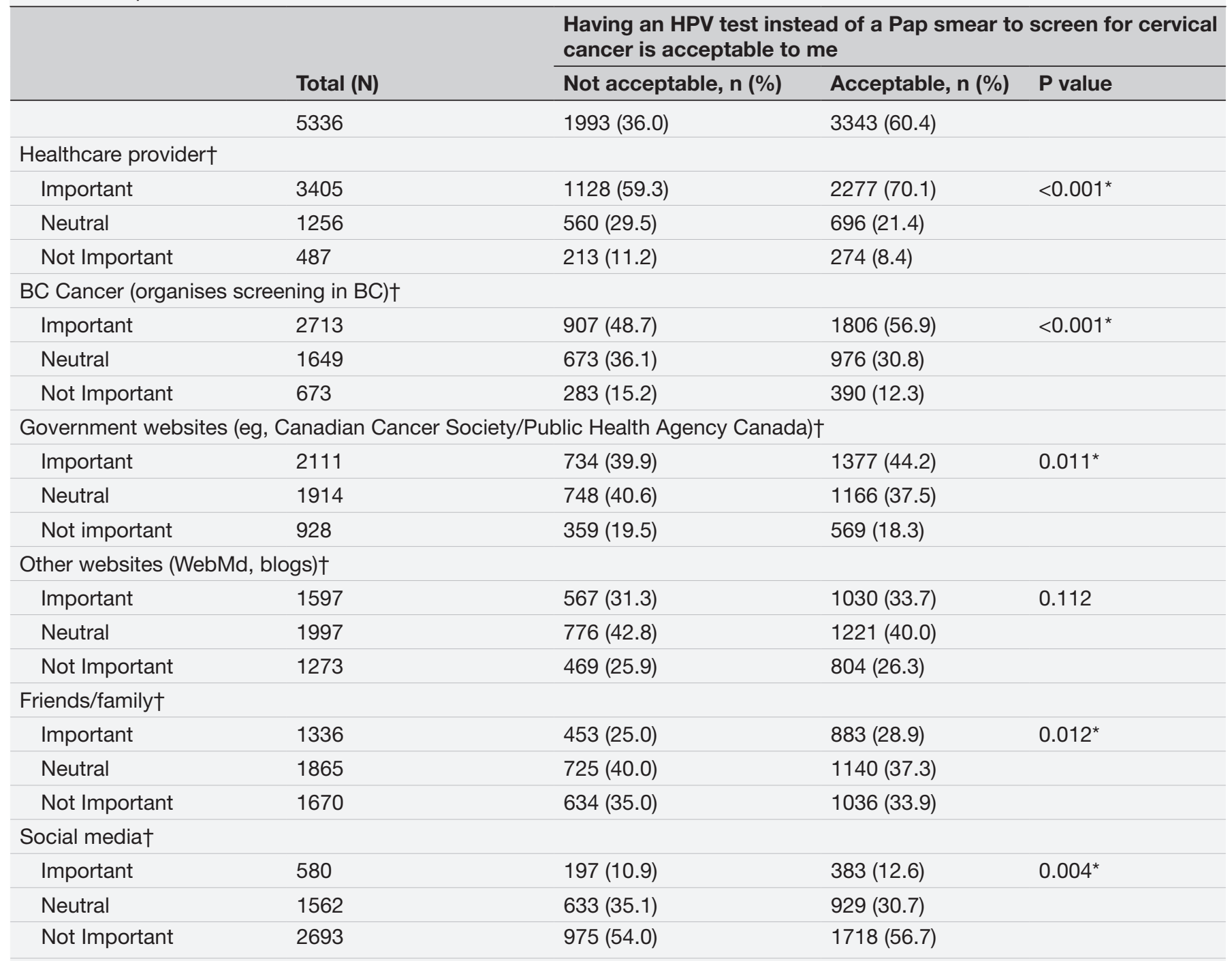

*Significant to $p<0.05$.

†Missing values up to $5336=$ not reported or prefer not to answer.

$\mathrm{BC}$, British Columbia; HPV, human papillomavirus.

\section{DISCUSSION}

Acceptability of, and attitudes towards primary HPV testing were analysed from 5532 women who completed HPV testing as part of the HPV FOCAL trial, which was embedded within an organised population-based cervical cancer screening programme. Most respondents (63\%) were accepting of HPV testing for cervix screening and for screening with HPV testing to begin at age 30 years $(69 \%)$. Just over half were accepting of HPV testing with the extended screen interval of $4-5$ years (54\%). Although most women were accepting, the proportion of respondents indicating they disagreed with or were neutral in their acceptance of HPV testing and of extended intervals was higher than we expected considering this was a group of people who were provided with education and information about HPV, HPV testing and cervical cancer. These findings are similar to other studies that indicate women have concerns about the extended interval recommended with HPV testing, ${ }^{10}$ 20-22 stemming from a belief that a cancer diagnosis may be missed through extension of the interval. Considering study participants received information regarding HPV, the natural history of HPV and cervical cancer and the rationale for HPV testing compared with the Pap test, these findings indicate that additional research and patient engagement is needed to gain insights and identify and develop resources or procedures to address barriers to HPV testing and an extended screening interval.

This multivariate analysis found that those who received positive HPV test results at some point during the trial were more likely to be accepting of HPV testing for cervix screening than those who never received an HPV positive result. Those who tested HPV positive would have received additional information and counselling from their healthcare provider and or a study nurse, which would not necessarily have been provided to those who 
tested HPV negative. This additional information would have reinforced the education participants were provided at trial baseline, including the prevalence of HPV in the population, the transient nature of HPV and the long natural history between HPV infection and cervical dysplasia development. In addition, those with HPV positive results would have received additional follow-up and management by the time the survey was administered. The reinforcement of education, an opportunity for dialogue when receiving the positive result, and the fact that those with a positive HPV result would have received treatment for detected dysplasia, may have facilitated improvement in knowledge and, subsequently, enhanced acceptance of HPV testing. Other findings have indicated that increased HPV and HPV screening knowledge can be a facilitator of HPV screening acceptance. ${ }^{18}$

One of the concerns with an extended screening interval is if women would be less likely to consult with the healthcare provider for other medical reasons. ${ }^{16}$ When we asked participants if they would be less willing to see a healthcare provider for other medical reasons if the interval for cervix screening were increased, most respondents said they would not be less willing, indicating that the extended interval for cervix screening would not prevent them from seeking care as needed. This finding can provide reassurance to healthcare professionals who have concerns that the extended screening interval recommended with HPV-based screening would lead to fewer visits to a clinician, given that the cervical screen visit is often an opportunity for the clinician to assess other preventive care or medical issues. ${ }^{23}$

Respondents' concerns regarding receipt of HPV positive results were varied. Nearly $93 \%$ of the respondents never received an HPV positive result during the HPV FOCAL trial. As a result, the majority of responses evaluate attitudes and experiences for those who did not actually receive HPV positive results, and therefore, reflect how the respondents would hypothetically feel if they were to receive HPV positive results.

Most participants indicated that having HPV would cause them concern about having cervical cancer. The majority of participants reported that having either an abnormal Pap or a positive HPV test would concern them; however, for those that had tested positive for HPV, they reported that an HPV test would concern them more compared with an abnormal Pap. Overall, participants' perceptions about HPV positive results and cervical cancer indicate that increased knowledge regarding the specificity of HPV testing for cervical cancer screening is needed.

Receipt of positive HPV results has been associated with higher anxiety and distress compared with receipt of abnormal Pap results, ${ }^{24-26}$ which may be due to the fact that HPV is an STI and has been associated with levels of shame and stigma. ${ }^{27} 28$ Most respondents in our survey indicated that receiving results for a sexually acquired HPV infection would concern them differently than having an abnormal Pap test result; however, this belief varied depending on a participant's acceptability of HPV testing. Women who were not accepting of HPV testing indicated that an HPV positive result would concern them differently than abnormal Pap results, compared with those who were accepting of HPV testing. Most respondents, whether they accepted HPV testing or not, felt it important for them to know who gave them HPV and when they got it (71\% and $78 \%$, respectively). These findings together are reflective of other research findings 92930 and indicate that when developing education and communication strategies, emphasis should be placed on the high prevalence of HPV in the population, the transient nature of most HPV infections and bringing awareness to the fact an infection may have been acquired several years prior to a positive test result. Differentiating HPV from other STIs may minimise anxiety and facilitate normalisation and acceptance. ${ }^{1627}$

Almost $75 \%$ of the respondents indicated they felt an HPV positive result would affect their relationship with their partner, or they were not sure, and almost $80 \%$ would be concerned about transmitting HPV to their partners, with many feeling they would be judged for being HPV positive. The stigma associated with HPV, concerns about infidelity and potential partner reactions to the HPV result may underlie these concerns. Previous research has indicated some women question whether partner notification with HPV is necessary. ${ }^{16}{ }^{31}$ Unlike other STIs such as chlamydia or gonorrhoea where partner notification is recommended for testing and treatment purposes, there is usually no medical reason to notify the partner of a woman who tested positive for HPV.

Healthcare providers, as trusted and valued sources of information, can influence patients' decision-making patterns regarding healthcare decisions. ${ }^{20}{ }^{32}$ Reflecting other findings, the respondents in this survey indicated that the most important sources of information for them were their healthcare providers and the provincial screening programme. ${ }^{20}{ }^{32}$ In this cohort, the least important sources of information were social media and friends and family, providing reassurance that women in this cohort seek information from reputable sources such as healthcare providers and the screening programme compared with the internet, or friends and family. These findings demonstrate that programme planning for HPVbased screening should prepare healthcare providers with adequate education and training surrounding HPV prior to programme changes, to ensure they are equipped to address women's questions and concerns regarding the paradigm shift from cytology to HPV-based screening.

This study is not without limitations. Survey participants were part of a large clinical trial and were given information about HPV, HPV testing and cervical cancer on enrolment and, therefore, may not be representative of all people eligible for cervix screening in British Columbia. However, participants of this study are reflective of the current population engaged in the screening programme, who receive cytology testing with the Pap smear and not HPV testing as standard of care. As a 
result, their concerns and feedback are informative for programmes planning for a shift from cytology to HPVbased screening. The response rate may be considered low at $42 \%$; however, those who have stronger opinions about their screening choices may have been more likely to respond to the survey than those who are more trusting of the healthcare system and accepting of any future policy changes. In addition, survey respondents were representative of overall participants in the HPV FOCAL trial. The majority of respondents completed the survey approximately 3 years after trial completion, and it is possible there was loss of recall of HPV related information provided to them when they consented to participate in the trial, which for many was up to 7 years prior to survey completion. The potential lag time between trial entry and survey completion may have introduced recall bias and impacted women's attitudes and beliefs surrounding HPV testing; however, the impact of this potential bias would be small as we found no significant difference between acceptability and time between trial entry and survey completion. In addition, most of the participants in this trial were over the age of 50 years, highly educated and primarily from two urban geographic regions and may not be representative of all screen eligible people in various regions of British Columbia.

\section{CONCLUSIONS}

In this study within an organised screening setting, evaluating acceptability and attitudes around HPV testing from women undergoing HPV-based screening, most are accepting of HPV testing for screening; however, further research is needed to understand factors that can increase acceptability. These findings contribute to the growing body of evidence demonstrating that concerns and experiences surrounding HPV testing and receipt of HPV positive results are complex and varied. As many cervix screening programmes begin HPV-based screening and are planning implementation strategies, attention to patient engagement to address potential barriers will be important. As HPV-based screening becomes standard of care, it is plausible that concerns with this paradigm shift will eventually be alleviated with increasing knowledge and familiarity. These findings provide insight into areas of importance that should be considered for development of education, information and communication strategies.

\footnotetext{
Author affiliations

${ }^{1}$ Department of Cancer Control Research, BC Cancer Agency, Vancouver, British Columbia, Canada

${ }^{2}$ Women's Health Research Institute, BC Women's Hospital and Health Centre, Vancouver, British Columbia, Canada

${ }^{3}$ Faculty of Medicine, The University of British Columbia, Vancouver, British

Columbia, Canada

${ }^{4}$ Department of Data and Analytics, BC Cancer Agency, Vancouver, British Columbia, Canada

${ }^{5}$ Public Health Laboratory, BC Centre for Disease Control, Vancouver, British

Columbia, Canada

${ }^{6}$ Cervix Screening Program, BC Cancer Agency, Vancouver, British Columbia, Canada
}

${ }^{7}$ Faculty of Health Sciences, Simon Fraser University, Burnaby, British Columbia, Canada

${ }^{8}$ Department of Oncology, McGill University, Montreal, Québec, Canada

Acknowledgements We would like to thank the thousands of women of British Columbia (BC) who participated in the HPV FOCAL trial and completed this survey and the hundreds of $\mathrm{BC}$ collaborating clinicians. We also extend gratitude to many partners including: The BC Cancer Cervix Screening and Colposcopy Program; the BC Centre for Disease Control Public Health Laboratory; the University of British Columbia; BC Cancer Surveillance and Outcomes Unit; Women's Health Research Institute; and the Canadian Institutes of Health Research.

Contributors LWS involved in conception, design and implementation of the research presented here and drafting and review of manuscript; CSR conducted statistical analysis and contributed to drafting and review of manuscript; LG conducted statistical analysis; MK, ML, REM, GS, SP, ELF and DvN are coinvestigators on the Human Papillomavirus For Cervical Cancer Screening Trial (HPV FOCAL) Study and were involved in FOCAL trial design and trial management; AJC and GSO are coprincipal investigators of the FOCAL Study and oversaw conduct of the trial; all authors take responsibility for the credibility of the data and analysis and critically reviewed the paper and approved the final version.

Funding This work was supported by the Canadian Institutes for Health Research. MCT-82072. CSR is supported by a Michael Smith Foundation for Health Research Fellowship.

Competing interests MK and AJC were principal investigators, and GSO, DvN and ELF were coinvestigators on industry-funded (Hologic Inc and Roche) investigatorled adjunct studies to the HPV FOCAL trial, designed to compare the performance of different HPV testing assays. No investigators personally benefitted financially. Funding for these adjunct studies was not applied to the operation of the HPV FOCAL results presented in this paper. ELF served as an occasional advisor for companies involved with HPV vaccines (Merck, GSK) and HPV diagnostics (Roche). He also holds a patent 'DNA methylation markers for early detection of cervical cancer', registered at the Office of Innovation and Partnerships, McGill University, Montreal, Quebec, Canada.

Patient consent for publication Consent obtained directly from patient(s)

Ethics approval Ethics approvals for survey was received by the University of British Columbia Research Ethics Board (H06-04032). In addition, a privacy review was undertaken to ensure the survey complied with provincial privacy legislation.

Provenance and peer review Not commissioned; externally peer reviewed.

Data availability statement Data are available on reasonable request. Deidentified participant data is available upon consideration and reasonable request.

Supplemental material This content has been supplied by the author(s). It has not been vetted by BMJ Publishing Group Limited (BMJ) and may not have been peer-reviewed. Any opinions or recommendations discussed are solely those of the author(s) and are not endorsed by BMJ. BMJ disclaims all liability and responsibility arising from any reliance placed on the content. Where the content includes any translated material, BMJ does not warrant the accuracy and reliability of the translations (including but not limited to local regulations, clinical guidelines, terminology, drug names and drug dosages), and is not responsible for any error and/or omissions arising from translation and adaptation or otherwise.

Open access This is an open access article distributed in accordance with the Creative Commons Attribution Non Commercial (CC BY-NC 4.0) license, which permits others to distribute, remix, adapt, build upon this work non-commercially, and license their derivative works on different terms, provided the original work is properly cited, appropriate credit is given, any changes made indicated, and the use is non-commercial. See: http://creativecommons.org/licenses/by-nc/4.0/.

ORCID iDs

Laurie W Smith http://orcid.org/0000-0002-9463-1713

Eduardo L Franco http://orcid.org/0000-0002-4409-8084

Gina S Ogilvie http://orcid.org/0000-0001-5783-4493

\section{REFERENCES}

1 Walboomers JM, Jacobs MV, Manos MM, et al. Human papillomavirus is a necessary cause of invasive cervical cancer worldwide. J Pathol 1999;189:12-19. 
2 Castellsagué X. Natural history and epidemiology of HPV infection and cervical cancer. Gynecol Oncol 2008;110:S4-7.

3 Ogilvie GS, van Niekerk D, Krajden M, et al. Effect of screening with primary cervical HPV testing vs cytology testing on highgrade cervical intraepithelial neoplasia at 48 months: the HPV focal randomized clinical trial. JAMA 2018;320:43-52.

4 Melnikow J, Henderson JT, Burda BU, et al. Screening for cervical cancer with high-risk human papillomavirus testing: updated evidence report and systematic review for the US preventive services Task force. JAMA 2018;320:687-705.

5 Ronco G, Dillner J, Elfström KM, et al. Efficacy of HPV-based screening for prevention of invasive cervical cancer: follow-up of four European randomised controlled trials. Lancet 2014;383:524-32.

6 Arbyn M, Ronco G, Anttila A, et al. Evidence regarding human papillomavirus testing in secondary prevention of cervical cancer. Vaccine 2012;30 Suppl 5:F88-99.

7 Dijkstra MG, van Zummeren M, Rozendaal L, et al. Safety of extending screening intervals beyond five years in cervical screening programmes with testing for high risk human papillomavirus: 14 year follow-up of population based randomised cohort in the Netherlands. BMJ 2016;355:i4924.

8 McBride E, Marlow LAV, Forster AS, et al. Anxiety and distress following receipt of results from routine HPV primary testing in cervical screening: the psychological impact of primary screening (PIPs) study. Int J Cancer 2020;146:2113-21.

9 Bennett KF, Waller J, Ryan M, et al. The psychosexual impact of testing positive for high-risk cervical human papillomavirus (HPV): a systematic review. Psychooncology 2019;28:1959-70.

10 Obermair HM, Dodd RH, Bonner C, et al. 'It has saved thousands of lives, so why change it?' content analysis of objections to cervical screening programme changes in Australia. BMJ Open 2018;8:e019171.

11 Ogilvie GS, van Niekerk DJ, Krajden M, et al. A randomized controlled trial of human papillomavirus (HPV) testing for cervical cancer screening: trial design and preliminary results (HPV focal trial). BMC Cancer 2010;10:111.

12 Ogilvie GS, Krajden M, van Niekerk DJ, et al. Primary cervical cancer screening with HPV testing compared with liquid-based cytology: results of round 1 of a randomised controlled trial -- the HPV focal study. Br J Cancer 2012;107:1917-24.

13 Coldman AJ, Gondara L, Smith LW, et al. Disease detection and resource use in the safety and control arms of the HPV focal cervical cancer screening trial. Br J Cancer 2016;115:1487-94.

14 Ogilvie GS, Krajden M, van Niekerk D, et al. Hpv for cervical cancer screening (HPV focal): complete round 1 results of a randomized trial comparing HPV-based primary screening to liquid-based cytology for cervical cancer. Int J Cancer 2017;140:440-8.

15 Ogilvie GS, Smith LW, van Niekerk DJ, et al. Women's intentions to receive cervical cancer screening with primary human papillomavirus testing. Int J Cancer 2013;133:2934-43.

16 Smith L, van Niekerk D, Coldman A, et al. Recommendations for implementing human Papillomavirus-Based cervical cancer screening: lessons learned from the HPV focal trial. J Obstet Gynaecol Can 2016;38:723-6.

17 The American Association for Public Opinion Research. Standard definitions: final dispositions of case codes and outcome rates for surveys. 9th Edition, 2016. https://www.aapor.org/AAPOR_Main/ media/publications/Standard-Definitions20169theditionfinal.pdf

18 Tatar O, Thompson E, Naz A, et al. Factors associated with human papillomavirus (HPV) test acceptability in primary screening for cervical cancer: a mixed methods research synthesis. Prev Med 2018;116:40-50.

19 Ogilvie GS, Smith LW, van Niekerk D, et al. Correlates of women's intentions to be screened for human papillomavirus for cervical cancer screening with an extended interval. BMC Public Health 2016;16.

20 Dodd RH, Mac OA, McCaffery KJ. Women's experiences of the renewed national cervical screening program in Australia 12 months following implementation: a qualitative study. BMJ Open 2020;10:e039041.

21 Silver MI, Rositch AF, Burke AE, et al. Patient concerns about human papillomavirus testing and 5-year intervals in routine cervical cancer screening. Obstet Gynecol 2015;125:317-29.

22 Gerend MA, Shepherd MA, Kaltz EA, et al. Understanding women's hesitancy to undergo less frequent cervical cancer screening. Prev Med 2017;95:96-102.

23 Saraiya M, Berkowitz Z, Yabroff KR, et al. Cervical cancer screening with both human papillomavirus and Papanicolaou testing vs Papanicolaou testing alone: what screening intervals are physicians recommending? Arch Intern Med 2010;170:977-86.

24 McBride E, Tatar O, Rosberger Z, et al. Emotional response to testing positive for human papillomavirus at cervical cancer screening: a mixed method systematic review with meta-analysis. Health Psychol Rev 2020:1-35.

25 Dodd $\mathrm{RH}$, Mac O, Brotherton JML, et al. Levels of anxiety and distress following receipt of positive screening tests in Australia's HPV-based cervical screening programme: a cross-sectional survey. Sex Transm Infect 2020;96:166-72.

26 McCaffery K, Waller J, Forrest S, et al. Testing positive for human papillomavirus in routine cervical screening: examination of psychosocial impact. BJOG 2004;111:1437-43.

27 Waller J, Marlow LAV, Wardle J. The association between knowledge of HPV and feelings of stigma, shame and anxiety. Sex Transm Infect 2007;83:155-9.

28 Shepherd MA, Gerend MA. The blame game: cervical cancer, knowledge of its link to human papillomavirus and stigma. Psychol Health 2014;29:94-109.

29 Kosenko KA, Hurley RJ, Harvey JA. Sources of the uncertainty experienced by women with HPV. Qual Health Res 2012;22:534-45.

30 Patel H, Moss EL, Sherman SM. Hpv primary cervical screening in England: women's awareness and attitudes. Psychooncology 2018:27:1559-64.

31 Bennett KF, Waller J, Ryan M. Concerns about disclosing a high-risk cervical human papillomavirus (HPV) infection to a sexual partner: a systematic review and thematic synthesis. BMJ Sexual and Reproductive Health 2019.

32 Rahman M, Laz TH, McGrath CJ, et al. Provider recommendation mediates the relationship between parental human papillomavirus (HPV) vaccine awareness and HPV vaccine initiation and completion among 13- to 17-year-old U.S. adolescent children. Clin Pediatr 2015;54:371-5 